\title{
A Estabilidade do Átomo de Hidrogênio Segundo a Eletrodinâmica Estocástica
}

The hydrogen atom stability after the Stochastic Electrodynamics

\author{
Roberto da Silva ${ }^{1}$, Humberto M. França ${ }^{2}$ \\ Universidade de São Paulo \\ ${ }^{1}$ Faculdade de Filosofia Ciências e Letras de Ribeirão Preto \\ Departamento de Física e Matemática Ribeirão Preto, SP, Brasil \\ ${ }^{2}$ Instítuto de Física, Departamento de Física Matemática, São Paulo, SP, Brasil
}

Recebido em 18 de dezembro, 2001. Aceito em 21 de janeiro, 2002.

\begin{abstract}
Neste trabalho evidenciamos a importância da radiação de ponto-zero para a estabilidade do átomo de hidrogênio do ponto de vista clássico. Nossos resultados estão baseados em trabalhos anteriores de T. H. Boyer e trata-se de uma revisão do trabalho por um dos autores [5], que usa eletromagnetismo clássico com a introdução de elementos estocásticos.
\end{abstract}

We discuss the importance of the zero-point radiation to the stability of the hydrogen atom from a classical perspective. Our review is based in previous work of Boyer on stochastic electrodynamics and in works of one of us [5] on the subject.

\section{Introdução}

Consideremos um elétron à temperatura $T=0$ (portanto sem radiação térmica) orbitando em torno de um próton. Através da fórmula de Larmor [1], sabemos que este elétron emite energia, por ser uma carga acelerada. Assim, devido a essa perda de energia por unidade de tempo, intuitivamente o que esperamos é que o elétron vá espiralado assintoticamente até colidir com o núcleo. Obviamente, isto é um absurdo, pois sabemos que o átomo de hidrogênio existe e é explicado satisfatoriamente pela Mecânica Quântica.

O objetivo deste trabalho é dar uma descrição da estabilidade do átomo de hidrôgenio, de forma que as leis do eletromagnetismo ainda sejam válidas, com a introdução de elementos estocásticos na análise clássica.

Para que um modelo clássico possa descrever o átomo de hidrogênio, um ingrediente fundamental é necessário; o estabelecimento da hipótese de que o elétron absorva uma energia do meio físico. Esta energia, proposta primordialmente por Planck e bastante discutida na década de 70 por T. H. Boyer (vide por exemplo[2]), estabeleceria então a possibilidade de existência de um raio estável para a órbita do elétron no átomo de de hidrogênio.

Neste artigo, pretendemos descrever em detalhes, baseados nos trabalhos de T. H. Boyer [2],[3],[4] e principalmente em [5], a obtenção deste raio através da eletrodinâmica estocástica com um ferramental matemático adequado a alunos de graduação de um curso de Física.

Concluiremos, fazendo uma conexão com a Mecânica Quântica, que este raio é exatamente o raio de Bohr, desde que admitamos a existência, no vácuo, de uma radiação aleatória atérmica de densidade espectral

$$
\rho_{0}(\omega)=\alpha \omega^{3}
$$

com $\alpha=\hbar / 2 \pi^{2} c^{3}$, onde $c$ é a velocidade da luz no vácuo e $\omega$ a frequência da radiação.

Para isso, o artigo segue este passo; na secção II, faremos uma breve dedução da potência de Larmor; na secção III, apresentamos uma modelagem estatística para a radiação de cavidade.

Nessa direção são considerados campos eletromagnéticos oscilantes, onde as fases são variáveis aleatórias. A seção termina com uma expressão fechada para a potência absorvida por um oscilador como função da densidade espectral associada à radiação térmica aleatória.

Finalmente, na secção IV, definimos a radiação de ponto-zero através de uma redefinição das flutuações térmicas e determinamos o raio de equilíbrio assim como sua estabilidade, mostrando que ele é exatamente o raio de Bohr:

$$
r_{e q}=\frac{\hbar^{2}}{m e^{2}} .
$$

Não há na literatura, no nosso entender, um texto dirigido para este resultado com o enfoque didático que aqui é apresentado. Cabe ressaltar que a relevância 
deste trabalho reside principalmente em fazer uma revisão da Ref. [5], 'lapidando' os resultados auxiliares necessários, com detalhes suficientes tal que alunos de graduação possam entender a importância da eletrodinâmica estocástica para a questão do átomo de hidrogênio. Isso também pode ser visto como uma forma para que professores de graduação possam enriquecer as aulas de eletromagnetismo usando um fato importantíssimo em Física, que é a compreensão de fenômenos aleatórios. Trata-se de um exemplo não trivial de aplicar conceitos estocásticos simples no Eletromagnetismo Clássico, disciplina extremamente importante na graduação em Física, obtendo-se um dos resultados essenciais da Mecânica Quântica que é o raio de Bohr, além da conclusão da sua estabilidade.

\section{A Potência Irradiada por uma carga acelerada: conceitos básicos.}

Um fenômeno bastante conhecido é que, quando uma carga é acelerada, ela emite radiação. Esta radiação é quantificada em termos da potência emitida pela carga acelerada.

Faremos, a seguir, uma breve dedução da potência emitida por uma carga acelerada, para o caso em que a velocidade da partícula é muito pequena quando comparada com a da luz $(v<<c)$, o que constitui o limite clássico não relativísitico.

Consideremos os campos de aceleração elétrico e magnético, aproximados no caso onde $v<<c$ (vide por exemplo [1]):

$$
\begin{aligned}
\vec{E}_{a} & =\frac{e}{c^{2} r^{2}}[\vec{r} \times(\vec{r} \times \vec{a})] \\
\vec{B}_{a} & =\frac{\vec{r} \times \vec{E}_{a}}{r}
\end{aligned}
$$

onde $c$ é a velocidade da luz, $e$ é a carga elementar, $\vec{a}$ é a aceleração e $\vec{r}$ é a posição de observação.

Usando a identidade vetorial $\vec{A} \times(\vec{B} \times \vec{C})=$ $\vec{B}(\vec{A} \cdot \vec{C})-\vec{C}(\vec{A} \cdot \vec{B})$, podemos concluir que

$$
E_{a}^{2}=\frac{e^{2} a^{2}}{c^{4} r^{2}} \sin ^{2} \theta
$$

onde $\theta$ é o ângulo entre os vetores $\vec{r}$ e $\vec{a}$.

A porção do vetor de Poynting que contribui para a radiação, em unidades de energia por unidade de tempo e por unidade de volume, é

$$
\begin{aligned}
\vec{S}_{a} & =\frac{c}{4 \pi}\left(\vec{E}_{a} \times \vec{B}_{a}\right)=\frac{c}{4 \pi} E_{a}^{2} \frac{\vec{r}}{r} \\
& =\frac{c}{4 \pi} E_{a}^{2} \vec{n}=\frac{e^{2} a^{2}}{4 \pi c^{3}} \frac{\sin ^{2} \theta}{r^{2}} \vec{n}
\end{aligned}
$$

O elemento de área de uma casca esférica, em coordenadas esféricas, é $d A=r^{2} \sin \theta d \theta d \varphi$ de modo que a potência irradiada por unidade de área é dada pelo módulo do vetor de Poynting:

$$
S_{a}=\frac{e^{2} a^{2}}{4 \pi c^{3}} \frac{\sin ^{2} \theta}{r^{2}}
$$

e através desta expressão, em termos de ângulo sólido $(d \Omega=\sin \theta d \theta d \varphi)$; podemos escrever que potência emitida na direção $\theta$ é:

$$
\frac{d P}{d \Omega}=\frac{e^{2} a^{2}}{4 \pi c^{3}} \sin ^{2} \theta
$$

Integrando sobre toda a superfície esférica, e sendo a aceleração centrípeta do elétron no átomo de hidrogênio, dada por $a=e^{2} / m r^{2}$, temos

$$
\begin{aligned}
P & =\frac{e^{2} a^{2}}{2 c^{3}} \int_{0}^{\pi} \sin ^{3} \theta d \theta \\
& =\frac{2}{3} \frac{e^{6}}{m^{2} c^{3} r^{4}} .
\end{aligned}
$$

Logo

$$
P \equiv P_{\text {Larmor }}=\frac{2}{3} \frac{e^{6}}{m^{2} c^{3} r^{4}} .
$$

Nesta expressão, $P_{\text {Larmor }}$ é definida como a potência de Larmor e representa qual é a potência emitida (ou dissipada) por uma carga acelerada que realiza uma órbita circular em um átomo de hidrogênio no limite clássico.

\section{Propriedades Estatísticas da Radiação de Cavidade}

\section{III.1 Um Modelo para a Radiação de Cavidade}

Como hipótese desse modelo, vamos representar nossa radiação em uma cavidade associando a cada ponto do espaço um campo elétrico aleatório e oscilante total dado pela soma (superposição) dos vários campos parciais $\vec{E}_{\vec{k}}(\vec{r}, t)$ : 


$$
\vec{E}(\vec{r}, t)=\sum_{k} \vec{E}_{\vec{k}}(\vec{r}, t)=\sum_{k} \sum_{\lambda=1}^{2} a_{T}(\vec{k}) \vec{\xi}(\vec{k}, \lambda) \cos \left(\vec{k} \cdot \vec{r}-\omega t+\theta_{\vec{k}, \lambda}\right),
$$

com a relação de dispersão $\omega=c k$. Aqui $a_{T}(\vec{k})$ é a amplitude de cada campo parcial cujo vetor de onda é $\vec{k}$, a uma dada temperatura $T$.

As duas possíveis polarizações associadas a cada $\vec{k}$ são $\vec{\xi}(\vec{k}, 1)$ e $\vec{\xi}(\vec{k}, 2)$. A aleatoriedade reside no fato de considerarmos as fases $\theta_{\vec{k}, \lambda}$ como sendo variáveis aleatórias estatisticamente independentes e uniformemente distribuídas no intervalo $[0,2 \pi]$.

\section{III.2 O Método de Einstein - Hopf}

Nesta subsecção, descreveremos o que se denomina por método de Einstein - Hopf, a fim de obter uma fórmula fechada para $a_{T}(\vec{k})$. A média nas fases aleatórias das componentes $x$ e $y$ de $\vec{E}$ é identicamente nula (veja equação 2)

$$
\left\langle E_{x}\right\rangle=\left\langle E_{y}\right\rangle \equiv \frac{1}{2 \pi} \sum_{k} \sum_{\lambda=1}^{2} a_{T} \xi_{y}(\vec{k}, \lambda) \int_{0}^{2 \pi} \cos \left(\vec{k} \cdot \vec{r}-\omega t+\theta_{k, \lambda}\right) d \theta_{k, \lambda}=0
$$

pois a integral de uma função periódica em todo seu período é nula. A soma sobre o índice $k$, deve ser entendida como uma soma sobre o vetor $\vec{k}$ (apenas uma simplificação de notação).

Fazendo $z=\vec{k} \cdot \vec{r}-\omega t$, na equação (3) e observando que

$$
\begin{aligned}
\int_{0}^{2 \pi} \cos \left(z+\theta_{k, \lambda}\right) d \theta_{k, \lambda} \int_{0}^{2 \pi} \cos \left(z^{\prime}+\theta_{k,,^{\prime} \lambda^{\prime}}\right) d \theta_{k^{\prime}, \lambda^{\prime}} & =\delta_{k k^{\prime}} \delta_{\lambda \lambda^{\prime}} \int_{0}^{2 \pi} d \theta_{k, \lambda} \cos ^{2}\left(z+\theta_{k, \lambda}\right) \\
& =\frac{1}{2} \delta_{k k^{\prime}} \delta_{\lambda \lambda^{\prime}}
\end{aligned}
$$

concluímos:

$$
\begin{aligned}
\left\langle E^{2}\right\rangle & =\frac{1}{2} \sum_{k} \sum_{\lambda=1}^{2} \sum_{k^{\prime}} \sum_{\lambda^{\prime}=1}^{2} a_{T}(\vec{k}) a_{T}\left(\overrightarrow{k^{\prime}}\right) \vec{\xi} \cdot \vec{\xi}^{\prime} \delta_{k k^{\prime}} \delta_{\lambda \lambda^{\prime}} \\
& =\frac{1}{2} \sum_{k} \sum_{\lambda=1}^{2} a_{T}^{2}(\vec{k}) \xi^{2}(\vec{k}, \lambda)
\end{aligned}
$$

Como a polarização é um versor, temos que $\sum_{\lambda=1}^{2} \xi^{2}(\vec{k}, \lambda)=1+1=2 ; \log \mathrm{o}$ :

$$
\left\langle E^{2}\right\rangle=\sum_{k} a_{T}^{2}(\vec{k})
$$

Do eletromagnetismo extraímos a informação de que a densidade média de energia de uma onda de radiação é $3 \cdot \frac{\left\langle E_{x}^{2}\right\rangle+\left\langle B_{x}^{2}\right\rangle}{8 \pi}$, onde $\left\langle E_{x}^{2}\right\rangle=\left\langle B_{x}^{2}\right\rangle$ para uma onda no vácuo, onde aqui $E_{x}$ é a componente $x$ do campo elétrico, e $B_{x}$ a respectiva componente do campo magnético associado à onda eletromagnética. Logo, se a densidade espectral na cavidade é $\rho(\omega, T)$, identifi- camos:

$$
\frac{3}{4 \pi}\left\langle E_{x}^{2}\right\rangle=\frac{\left\langle E^{2}\right\rangle}{4 \pi}=\int_{0}^{\infty} \rho(\omega, T) d \omega
$$

Conforme é mostrado por Boyer [3], a decomposição de Fourier (ver equação 2) é tal que $\sum_{k} \rightarrow \frac{V}{(2 \pi)^{3}} \int d^{3} k$, onde $V$ é um volume característico. Este volume será eliminado mais adiante. Da equação (4) vem:

$$
\begin{aligned}
\left\langle E^{2}\right\rangle & =\frac{V}{(2 \pi)^{3}} \int a_{T}^{2}(\omega) d^{3} k \\
& =4 \pi \frac{V}{(2 \pi)^{3}} \int_{0}^{\infty} k^{2} a_{T}^{2}(\omega) d k
\end{aligned}
$$

Como $k=\frac{\omega}{c}$, temos

$$
\left\langle E^{2}\right\rangle=4 \pi \frac{V}{(2 \pi c)^{3}} \int_{0}^{\infty} \omega^{2} a_{T}^{2}(\omega) d \omega
$$

Comparando-se (6) com (5) tem-se que:

$$
a_{T}(\omega)=\left[\frac{(2 \pi c)^{3} \rho(\omega, T)}{\omega^{2} V}\right]^{1 / 2}
$$




\section{III.3 Oscilador harmônico em equilibrio com a radiação térmica aleatória}

Mostra-se que a componente $x$ da força de radiação ${ }^{1}$ é dada por

$$
F_{r} \approx \frac{2}{3} \frac{e^{2}}{c^{3}} \dddot{x},
$$

(para uma demonstração desta fórmula vide por exemplo[1]).

Assim, escrevemos a segunda lei de Newton de um oscilador harmônico em equilíbrio com a radiação térmica:

$$
\ddot{x}=-\omega_{0}^{2} x+\frac{2}{3} \frac{e^{2}}{c^{3}} \dddot{x}+\frac{e}{m} E_{x}(t) .
$$

Em primeira aproximação temos que $\dddot{x}=$ $-\omega_{0}^{2} x \Rightarrow \dddot{x}=-\omega_{0}^{2} \dot{x}$. Logo, desse resultado em (8) obtemos

$$
\ddot{x}=-\omega_{0}^{2} x-\gamma \dot{x}+\frac{e}{m} E_{x}(t)
$$

onde $\gamma \equiv \frac{2}{3} \frac{e^{2} \omega_{0}^{2}}{c^{3}}$. Sendo que

$$
E_{x}=\frac{1}{2 \pi} \sum_{k} \sum_{\lambda=1}^{2} a_{T} \xi_{x}(\vec{k}, \lambda) \cos \left(\vec{k} \cdot \vec{r}-\omega t+\theta_{k, \lambda}\right)
$$

por substituição direta demonstra-se facilmente que

$$
x(t)=\frac{e}{2 m} \sum_{k} \sum_{\lambda=1}^{2} a_{T} \xi_{x}(\vec{k}, \lambda)\left[\frac{\exp \left[\vec{k} \cdot \vec{r}-i \omega t+\theta_{k, \lambda}\right]}{D(\omega)}+\frac{\exp \left[\vec{k} \cdot \vec{r}-i \omega t+\theta_{k, \lambda}\right]}{D^{*}(\omega)}\right]
$$

é solução de $(9)$, onde $D(\omega)=\omega_{0}^{2}-\omega^{2}-i \gamma \omega$ e $D^{*}$ seu complexo conjugado.

\section{III.4 Potência média absorvida por um oscilador sob radiação térmica aleatória}

Nosso objetivo agora é obter a potência absorvida por um oscilador harmônico. Como estamos lidando com grandezas estocásticas, estamos interessa- dos na potência média. Podemos nos convencer facilmente de que a potência média absorvida por um oscilador harmônico, diante das grandezas já previamente definidas é:

$$
P_{a b s}=\left\langle\dot{x} e E_{x}+\dot{y} e E_{y}\right\rangle .
$$

Assim de (11) pode-se concluir que

$$
\dot{x}(t)=\frac{e}{2 m} \sum_{k} \sum_{\lambda=1}^{2} a_{T} \xi_{x}(\vec{k}, \lambda)\left[-i \omega \frac{\exp \left[\vec{k} \cdot \vec{r}-i \omega t+\theta_{k, \lambda}\right]}{D(\omega)}+i \omega \frac{\exp \left[\vec{k} \cdot \vec{r}-i \omega t+\theta_{k, \lambda}\right]}{D^{*}(\omega)}\right] .
$$

E desta forma

$$
P_{a b s}=\frac{e^{2}}{4 m} \sum_{k} \sum_{\lambda=1}^{2} \sum_{k^{\prime}} \sum_{\lambda^{\prime}=1}^{2} a_{T}(\omega) a_{T}\left(\omega^{\prime}\right) \xi_{x} \xi_{x}^{\prime}\left\langle f\left(\omega, \omega^{\prime}, \theta_{k, \lambda}, \theta_{k^{\prime}, \lambda^{\prime}}\right)\right\rangle
$$

com

$$
\begin{aligned}
\langle f\rangle=\left\langle\left(-i \omega \frac{\exp \left[\vec{k} \cdot \vec{r}-i \omega t+\theta_{k, \lambda}\right]}{D(\omega)}+i \omega \frac{\exp \left[\vec{k} \cdot \vec{r}-i \omega t+\theta_{k, \lambda}\right]}{D^{*}(\omega)}\right) .\right. \\
\left.\cdot\left(\exp \left[\overrightarrow{k^{\prime}} \cdot \vec{r}-i \omega^{\prime} t+\theta_{k^{\prime}, \lambda^{\prime}}\right]+\exp \left[-\overrightarrow{k^{\prime}} \cdot \vec{r}+i \omega^{\prime} t-\theta_{k^{\prime}, \lambda^{\prime}}\right]\right)\right\rangle
\end{aligned}
$$

Percebemos que, no cálculo de $\langle f\rangle$, aparecem as seguintes integrais:

$$
\left\langle e^{i \theta_{k, \lambda}} e^{i \theta_{k^{\prime}, \lambda^{\prime}}}\right\rangle=\frac{1}{(2 \pi)^{2}} \int_{0}^{2 \pi} e^{i \theta_{k, \lambda}} d \theta_{k, \lambda} \int_{0}^{2 \pi} e^{i \theta_{k^{\prime}, \lambda^{\prime}}} d \theta_{k^{\prime}, \lambda^{\prime}}=0,
$$

\footnotetext{
${ }^{1}$ Escolhemos $x$ sem nenhuma perda de generalidade.
} 
e, conseqüentemente

$$
\left\langle e^{i \theta_{k, \lambda}} e^{-i \theta_{k^{\prime}, \lambda^{\prime}}}\right\rangle=\left\{\begin{array}{l}
0, \text { se } k^{\prime} \neq k \text { e } \lambda \neq \lambda^{\prime} \\
\frac{1}{(2 \pi)^{2}}\left[\int_{0}^{2 \pi} d \theta\right]^{2}=1, \text { se } k^{\prime}=k \text { e } \lambda=\lambda^{\prime}
\end{array} .\right.
$$

Assim, de (13), com o auxílio de (14) e (15), obtemos que

$$
P_{a b s}=\frac{e^{2}}{2 m} \sum_{k} \sum_{\lambda=1}^{2} a_{T}^{2}(\omega) \xi_{x}^{2} \frac{\gamma \omega^{2}}{\left(\omega_{0}^{2}-\omega^{2}\right)^{2}+\gamma^{2} \omega^{2}}
$$

No apêndice deste texto, é provado que

$$
\sum_{\lambda=1}^{2} \xi_{i}(\vec{k}, \lambda) \xi_{j}(\vec{k}, \lambda)=\delta_{i j}-\frac{k_{i} k_{j}}{k^{2}}
$$

Em posse deste fato, escrevemos

$$
\sum_{\lambda=1}^{2} \xi_{x}^{2}(k, \lambda)=1-\frac{k_{x}^{2}}{k^{2}}
$$

Substituindo-se (16) em (18), conclui-se

$$
P_{a b s}=\frac{e^{2}}{m} \sum_{k} a_{T}^{2}(\omega)\left(1-\frac{k_{x}^{2}}{k^{2}}\right) \frac{\gamma \omega^{2}}{\left(\omega_{0}^{2}-\omega^{2}\right)^{2}+\gamma^{2} \omega^{2}}
$$

Observando que

$$
\int \frac{k_{x}^{2}+k_{y}^{2}+k_{z}^{2}}{k^{2}} d \Omega=\int d \Omega=4 \pi
$$

temos por simetria que $\int \frac{k_{x}^{2}}{k^{2}} d \Omega=\frac{4}{3} \pi$.

Assim, usando o procedimento $\sum_{k} \rightarrow \frac{V}{(2 \pi)^{3}} \int d^{3} k$ e com $\omega=k c$ de (19), concluímos

$$
P_{a b s}=\frac{8}{3} \frac{e^{2}}{m} \frac{V}{(2 \pi)^{3}} \frac{\pi}{c^{3}} \int_{0}^{\infty} \frac{\gamma \omega^{4} a_{T}^{2}(\omega)}{\left(\omega_{0}^{2}-\omega^{2}\right)^{2}+\gamma^{2} \omega^{2}} d \omega
$$

Substituindo (7) em (20), temos

$$
P_{a b s}=\frac{8}{3} \frac{\pi \gamma}{m} e^{2} \int_{0}^{\infty} \frac{\omega^{2} \rho(\omega, T)}{\left(\omega_{0}^{2}-\omega^{2}\right)^{2}+\gamma^{2} \omega^{2}} d \omega
$$

Expandindo $\rho(\omega, T)$ em torno da frequência $\omega_{0}$, onde $\rho$ tem um máximo pronunciado, $\rho$ só deve contribuir numa pequena vizinhança de $\omega_{0}$, e em primeira aproximação temos que $\rho(\omega, T) \approx \rho\left(\omega_{0}, T\right)$. Supondo também que o integrando varie lentamente na região de ressonância, temos que

$$
\int_{0}^{\infty} \frac{\omega^{2}}{\left(\omega_{0}^{2}-\omega^{2}\right)^{2}+\gamma^{2} \omega^{2}} d \omega \approx \frac{1}{4} \int_{0}^{\infty} \frac{1}{\left(\omega_{0}-\omega\right)^{2}+(\gamma / 2)^{2}} d \omega
$$

Fazendo-se a mudança de variáveis $\omega_{0}-\omega=-\frac{\gamma x}{2}$, e sabendo-se que $\omega_{0} \gg \gamma$ tem-se:

$$
\begin{aligned}
\int_{0}^{\infty} \frac{\omega^{2}}{\left(\omega_{0}^{2}-\omega^{2}\right)^{2}+\gamma^{2} \omega^{2}} d \omega & \approx \frac{1}{2 \gamma^{2}} \int_{\frac{-2 \omega_{0}}{\gamma}}^{\infty} \frac{\gamma}{x^{2}+1} d x \\
& \approx \frac{1}{2 \gamma} \int_{-\infty}^{\infty} \frac{1}{x^{2}+1} d x \\
& =\frac{\pi}{2 \gamma}
\end{aligned}
$$

Após todas essas considerações a equação (21) toma a forma

$$
P_{a b s}=\frac{4}{3} \frac{\pi^{2}}{m} e^{2} \rho\left(\omega_{0}, T\right)
$$

\section{A radiação de ponto-zero e a fórmula de Planck para o es- pectro do corpo negro.}

\section{IV.1 A Lei de Wien, e o surgimento da radiação de ponto-zero}

A lei de Wien, que é baseada em argumentos físicos puramente clássicos, pode ser formulada através da seguinte fórmula para densidade espectral:

$$
\rho(\omega, T)=\omega^{3} \Phi(\omega / T)
$$

onde $\Phi(\omega / T)$ tem a única exigência de ser uma função derivável. Desta equação nós conseguimos derivar facilmente a equação de Fermi:

$$
T \frac{\partial \rho}{\partial T}=3 \rho-\omega \frac{\partial \rho}{\partial \omega}
$$

Para $T=0$, encontramos duas possíveis soluções para esta equação: uma trivial $\rho_{0}(\omega)=0$ e a outra $\rho_{0}(\omega)=\alpha \omega^{3}$. Podemos postular que, na cavidade, a densidade espectral total é

$$
\rho(\omega, T)=\alpha \omega^{3}+\rho_{T}(\omega)
$$

onde $\rho_{T}(\omega)$ tem origem térmica e $\rho_{0}(\omega)=\alpha \omega^{3}$ é a chamada radiação de ponto-zero de forma que quando $T=0$ :

$$
\rho(\omega, T=0)=\rho_{0}(\omega)=\alpha \omega^{3} ;
$$

e então só radiação de ponto-zero é que existe. Assim, a temperatura nula, conclui-se de (22) que

$$
P_{a b s}(T=0)=\frac{4}{3} \frac{\pi^{2} \alpha}{m} e^{2} \omega^{3}
$$


A constante $\alpha$ será fixada mais adiante por comparação com dados experimentais.

\section{IV.2 Dedução clássica do espectro de corpo negro}

Usando-se exatamente os mesmos argumentos empregados para calcular $\left\langle e \dot{x} E_{x}\right\rangle$, obtém-se

$$
\left\langle x^{2}\right\rangle=\frac{4}{3} \frac{\pi e^{2}}{m^{2}} \rho\left(\omega_{0}, T\right) \int_{0}^{\infty} \frac{1}{\left(\omega_{0}^{2}-\omega^{2}\right)^{2}+\gamma^{2} \omega^{2}} d \omega
$$

da mesma maneira como feito no caso de $\left\langle e \dot{x} E_{x}\right\rangle$. Analogamente, fazendo a mudança de variáveis $\omega_{0}-\omega=$ $-\frac{\gamma x}{2}$, e sendo que $\omega_{0} \gg \gamma$ tem-se

$$
\left\langle x^{2}\right\rangle=\frac{4}{3} \frac{\pi e^{2}}{m^{2}} \frac{\pi \rho\left(\omega_{0}, T\right)}{2 \gamma \omega_{0}^{2}}
$$

Sendo a energia média dada por

$$
\langle\varepsilon\rangle=\frac{1}{2} m\left(\left\langle\dot{x}^{2}\right\rangle+\omega_{0}^{2}\left\langle x^{2}\right\rangle\right)
$$

visto que (11) e (12) implicam que $\left\langle\dot{x}^{2}\right\rangle=\omega_{0}^{2}\left\langle x^{2}\right\rangle$. Como $\langle\varepsilon\rangle=k T$, concluímos que

$$
\langle\varepsilon\rangle=\frac{\pi^{2} c^{3} \rho\left(\omega_{0}, T\right)}{\omega_{0}^{2}}=k T
$$

Notemos que, sem energia de ponto-zero, nós temos uma determinada definição de temperatura, ou de flutuação térmica, dada pela equação diferencial

$$
k T^{2} \frac{\partial\langle\varepsilon\rangle}{\partial T}=\langle\varepsilon\rangle^{2}
$$

$\operatorname{com}\langle\varepsilon\rangle=k T$. Com a radiação de ponto-zero, temos que redefinir a flutuação total em $\langle\varepsilon\rangle$ de forma que

$$
k T^{2} \frac{\partial\langle\varepsilon\rangle}{\partial T}=\langle\varepsilon\rangle^{2}-\left\langle\varepsilon_{0}\right\rangle^{2}
$$

com

$$
\langle\varepsilon\rangle=\frac{\pi^{2} c^{3}}{\omega_{0}^{2}}\left[\alpha \omega_{0}^{3}+\rho_{T}\left(\omega_{0}\right)\right]=\left\langle\varepsilon_{0}\right\rangle+\left\langle\varepsilon_{T}\right\rangle
$$

onde $\left\langle\varepsilon_{0}\right\rangle=\pi^{2} c^{3} \alpha \omega_{0}$.

Usando o resultado da integral:

$$
\int \frac{1}{a^{2}-b^{2} x^{2}} d x=\frac{1}{2 a b} \ln \left(\left|\frac{a+b x}{a-b x}\right|\right)
$$

obtemos da equação (24), após sua integração, que

$$
A+\frac{1}{k T}=\frac{1}{2\left\langle\varepsilon_{0}\right\rangle} \ln \left(\frac{\langle\varepsilon\rangle+\left\langle\varepsilon_{0}\right\rangle}{\langle\varepsilon\rangle-\left\langle\varepsilon_{0}\right\rangle}\right)
$$

onde $A$ é uma constante de integração. No limite $T \rightarrow \infty,\langle\varepsilon\rangle \gg\left\langle\varepsilon_{0}\right\rangle$ e $A=0$. Obtém-se, então

$$
\langle\varepsilon\rangle=\left\langle\varepsilon_{0}\right\rangle\left[1+\frac{2}{\exp \left[\frac{2\left\langle\varepsilon_{0}\right\rangle}{k T}\right]-1}\right]
$$

Conclui-se deste resultado e da equação (25), uma expressão para densidade espectral

$$
\rho_{T}\left(\omega_{0}\right)=\frac{2 \alpha \omega_{0}^{3}}{\exp \left[\frac{2 \pi^{2} c \alpha \omega_{0}}{k T}\right]-1},
$$

que, quando comparada com a fórmula da radiação de Planck da mecânica quântica, a saber

$$
\rho_{T}(\omega)=\frac{\hbar \omega^{3}}{\pi^{2} c^{3}} \frac{1}{\left(e^{\frac{\hbar \omega}{k T}}-1\right)}
$$

fornece a relação que conecta a constante $\alpha$ com a constante de Planck:

$$
2 \pi^{2} c^{3} \alpha=\hbar
$$

Assim, da equação (23), escrevemos a fórmula explícita da potência absorvida a temperatura nula como função da constante de Planck

$$
P_{a b s}(T=0) \approx \frac{2}{3} \frac{\hbar e^{5}}{m^{5 / 2} c^{3} r^{9 / 2}}
$$

onde ${ }^{2}$ foi feito $\omega^{2}=e^{2} / m r^{3}$

\section{IV.3 Raio de equilíbrio do átomo de hidrogênio via mecânica clássica}

A energia total do elétron no átomo de hidrogênio é dada por

$$
\varepsilon=K+V=-\frac{e^{2}}{2 r}
$$

A potência total deste sistema é a taxa de variação da energia pelo tempo, isto é, a potência total, que é a diferença entre a potência absorvida e a potência dissipada (de Larmor). Assim, de acordo com as equações (1) e (26), a $T=0$ :

$$
\begin{aligned}
\frac{d}{d t}\left(-\frac{e^{2}}{2 r}\right) & =P_{a b s}-P_{\text {Larmor }} \\
& =\frac{2}{3} \frac{e^{6}}{m^{2} c^{3} r^{4}}\left[\frac{\hbar}{e(m r)^{1 / 2}}-1\right],
\end{aligned}
$$

obtendo a equação diferencial

$$
\frac{e^{2} \dot{r}}{2 r^{2}}=\frac{2}{3} \frac{e^{6}}{m^{2} c^{3} r^{4}}\left[\frac{\hbar}{e(m r)^{1 / 2}}-1\right]
$$

Exigindo que $\dot{r}=0$ nesta equação determinamos o raio de equilíbrio

\footnotetext{
${ }^{2}$ Isto é obtido facilmente igualando-se a força centrípeta com a força coulombiana entre o elétron e o próton no átomo de hidrogênio.
} 


$$
r_{e q}=\frac{\hbar^{2}}{m e^{2}}
$$

observando-se que este raio é exatamente o raio de Bohr. A solução exata da equação (27) fornece uma órbita espiral conforme foi recentemente discutido $[5,6]$.

Vamos mostrar que esse raio é estável. Os comportamentos assintóticos de $P_{a b s}$ e $P_{\text {Larmor }}$ para $\mathrm{r}$ grande $(r \rightarrow \infty)$, são $\frac{1}{r^{9 / 2}}$ e $\frac{1}{r^{4}}$ respectivamente. Isso nos remete às condições:

$$
\frac{d P_{\text {Larmor }}}{d r}<0 \text { e } \frac{d^{2} P_{\text {Larmor }}}{d r^{2}}>0
$$

e

$$
\frac{d P_{a b s}}{d r}<0 \text { e } \frac{d^{2} P_{a b s}}{d r^{2}}>0
$$

E com isso podemos dizer que ambas as funções $P_{\text {Larmor }}$ e $P_{\text {abs }}$ decrescem com concavidade voltada para baixo. Então, com isso, podemos concluir que se $r>r_{e q}, P_{\text {Larmor }}>P_{a b s}$, o elétron perde energia, e a tendência é retornar ao raio de equilíbrio. Analogamente para $r<r_{e q}, P_{\text {Larmor }}<P_{a b s}$, o elétron ganha energia, e a tendência é retornar ao raio de equilíbrio. Concluímos que o raio de equilíbrio é estável mesmo a temperatura nula. É bastante instrutivo comparar esta discussão clássica da estabilidade do átomo com aquela válida na Eletrodinâmica Quântica [7]. Conforme discutido nas referências [5] e [7] vemos que é precisamente a radiação de ponto-zero do vácuo a fonte de energia responsável pela estabilidade do estado fundamental de sistemas microscópicos.

\section{Apêndice.}

\section{V.1 Dedução da fórmula (17)}

$\operatorname{Vamos} \operatorname{associar} \vec{\xi}(\vec{k}, 1) \rightarrow \overrightarrow{e_{r}}, \vec{\xi}(\vec{k}, 2) \rightarrow \overrightarrow{e_{\varphi}}$, e $\vec{k} \rightarrow$ $k \overrightarrow{e_{\theta}}$ num sistema de coordenadas polares. Consideremos dois casos e outros podem ser obtidos por analogia.

Caso $1(i=j=x)$

$$
\begin{gathered}
\vec{\xi}(\vec{k}, 1) \\
\xi_{x}(\vec{k}, 1)=\vec{\xi}(\vec{k}, 1) \cdot \vec{e}_{x}=\vec{e}_{r} \cdot \vec{e}_{x}=\sin \theta \cos \varphi \\
\xi_{x}(\vec{k}, 2)=\vec{\xi}(\vec{k}, 2) \cdot \vec{e}_{x}=\vec{e}_{\varphi} \cdot \vec{e}_{x}=-\sin \varphi
\end{gathered}
$$

Portanto

$$
\sum_{\lambda=1}^{2} \xi_{x}^{2}(\vec{k}, \lambda)=\sin ^{2} \theta \cos ^{2} \varphi+\sin ^{2} \varphi
$$

Por outro lado

$$
\delta_{x x}-\frac{k_{x} k_{x}}{k^{2}}=1-\frac{k^{2} \cos ^{2} \theta \cos ^{2} \varphi}{k^{2}}=\sin ^{2} \varphi+\sin ^{2} \theta \cos ^{2} \varphi
$$

De forma que podemos concluir para este caso que

$$
\sum_{\lambda=1}^{2} \xi_{x}^{2}(\vec{k}, \lambda)=\delta_{x x}-\frac{k_{x} k_{x}}{k^{2}}
$$

Caso $2(i=x, j=y, \operatorname{com} x \neq y)$

$$
\begin{aligned}
& \xi_{x}(\vec{k}, 1)=\sin \theta \cos \varphi \\
& \xi_{x}(\vec{k}, 2)=-\sin \varphi \\
& \xi_{y}(\vec{k}, 1)=\sin \theta \sin \varphi \\
& \xi_{y}(\vec{k}, 2)=\cos \varphi
\end{aligned}
$$

Portanto

$$
\sum_{\lambda=1}^{2} \xi_{x}(\vec{k}, \lambda) \xi_{y}(\vec{k}, \lambda)=-\sin \varphi \cos \varphi \cos ^{2} \theta
$$

Por outro lado $\delta_{x y}=0$, e portanto

$$
\delta_{x y}-\frac{k_{x} k_{y}}{k^{2}}=-\cos ^{2} \theta \sin \varphi \cos \varphi .
$$

Com isso concluímos que $\sum_{\lambda=1}^{2} \xi_{x}(\vec{k}, \lambda) \xi_{y}(\vec{k}, \lambda)=\delta_{x y}-$ $\frac{k_{x} k_{y}}{k^{2}}$ Os outros casos são idênticos.

\section{Agradecimentos}

R. da Silva agradece a FAPESP pelo apoio financeiro e L. Junges Subtil por suas apreciações sobre este texto.

\section{Referências}

[1] Princípios de Eletrodinâmica Clássica, J. Frenkel, Edusp.

[2] T. H. Boyer, Classical Statistical Thermodynamics and Eletromagnetic Zero-Point Radiation, Phys. Rev. 186, 1304 (1969).

[3] T. H. Boyer, Random Electrodynamics: The theory of classical electrodynamics with classical electromagnetic zero-point radiation, Phys Rev D 11, 790 (1975).

[4] T. H. Boyer, General Connection between random electrodynamics and quantum electrodynamics for free electromagnetic fields and for dipole oscillator systems, Phys. Rev. D 11, 909 (1975).

[5] H. M. França, H. Franco and C. P. Malta, A stochastic electrodynamics interpretation of spontaneous transitions in the hydrogen atom, Eur. J. Phys. 18, 343 (1997).

[6] M. Seidl and P. O. Lipas, Semiclassical interpretation of spontaneous transitions in the hydrogen atom, Eur. J. Phys. 17, 25 (1996).

[7] J. Dalibard, J. Dupont-Roc and C. Cohen-Tannouji, Vacuum fluctuations and radiation reaction: identification of their respective contributions, J. Physique 43, 1617 (1982). 